\title{
A NOTE ON EXTENDING SEMICHARACTERS ON SEMIGROUPS
}

\section{KENNETH A. ROSS ${ }^{1}$}

The purpose of this note is to give a necessary and sufficient condition for a semicharacter on a subsemigroup of a commutative semigroup $G$ to be extendable to a semicharacter on $G$. A bounded complex function $\chi$ on a semigroup $G$ is called a semicharacter of $G$ if $\chi(x) \neq 0$ for some $x \in G$ and $\chi(x y)=\chi(x) \chi(y)$ for all $x, y \in G$. Semicharacters were introduced by Hewitt and Zuckerman [1] and in a slightly different form by $\breve{S}$. Schwarz, [4] and [5]. See also [2]. If $\chi$ is a semicharacter on $G$, then $|\chi(x)| \leqq 1$ for all $x \in G$. We will write $a \mid b$ if there exists an $x \in G$ such that $a x=b$.

We note that our theorem generalizes [6, Lemma 1, p. 94], and is related to [3, Lemma 1 , p. 364]. Some remarks on the impossibility of generalizing our theorem appear at the end of the paper.

Theorem. Let $G$ be a commutative semigroup and let $S \subseteq G$ be a subsemigroup. Then if $\chi$ is a semicharacter on $S$, the condition

$$
a \mid b \text { and } a, b \in S \text { imply }|\chi(a)| \geqq|\chi(b)| \text {, }
$$

is necessary and sufficient for $\chi$ to be extendable to a semicharacter on $G$.

Proof. The necessity of (A) is clear. In proving the sufficiency, we first show that we may suppose that $G$ has a unit. For suppose that the theorem is known for semigroups with unit. Then if $G$ is a semigroup without unit, we adjoint a unit in the manner of $[1$, Theorem 2.3] and then apply the known result. The restriction to $G$ of the semicharacter so obtained is the desired semicharacter. In the remainder of the proof it will be supposed that $G$ has a unit $e$.

Let $a$ consist of all pairs $(\tilde{\chi}, T)$ such that

(1) $S \subseteq T$ and $T$ is a subsemigroup of $G$,

(2) $\tilde{\chi}$ is a semicharacter on $T$,

(3) $\tilde{\chi}$ satisfies (A) on $T$, and

(4) $\tilde{\chi}$ extends $\chi$.

We say that $\left(\tilde{\chi}_{1}, T_{1}\right) \leqq\left(\tilde{\chi}_{2}, T_{2}\right)$ if $T_{1} \subseteq T_{2}$ and $\tilde{\chi}_{2}$ extends $\tilde{\chi}_{1}$. By Zorn's lemma $Q$ has a maximal element $\left(\psi, T_{0}\right)$. Clearly $e \in T_{0}$ for otherwise $\psi$ can be trivially extended to the subsemigroup $T_{0} \cup\{e\}$. If $T_{0}=G$, the proof is complete. Suppose then that $T_{0} \subset G$. In each of the follow-

Received by the editors November 17, 1958.

${ }^{1}$ Supported by a National Science Foundation pre-doctoral fellowship. The writer is indebted to Professor Edwin Hewitt for valuable advice. 
ing cases we will choose an $x_{0} \in G-T_{0}$ and then extend $\psi$ to a semicharacter $\tilde{\psi}$ on the subsemigroup $T_{1}=\left\{t x_{0}^{k}: t \in T_{0}, k \geqq 0\right\}$ such that $\tilde{\psi}$ satisfies $(\mathrm{A})$ on $T_{1}$. Since $T_{1} \supset T_{0}$, this will constitute a contradiction.

CAse 1. Suppose there exist $a_{0}, b_{0} \in T_{0}$ and $x_{0} \in G-T_{0}$ such that $a_{0} x_{0}=b_{0}$ and $\psi\left(b_{0}\right) \neq 0$.

By (A) applied to $T_{0}, \psi\left(a_{0}\right) \neq 0$. Let $\tilde{\psi}\left(t x_{0}^{k}\right)=\psi(t) \psi\left(b_{0}\right)^{k} / \psi\left(a_{0}\right)^{k}$ for $t x_{0}^{k} \in T_{1}$. Suppose that $t x_{0}^{k}=u x_{0}^{l}$, where $t, u \in T_{0}$. Then $a_{0}^{k+l} t x_{0}^{k}=a_{0}^{k+l} u x_{0}^{l}$ and so $b_{0}^{k} a_{0}^{l} t=b_{0}^{l} a_{0}^{k} u$. Hence

$$
\tilde{\psi}\left(t x_{0}^{k}\right)=\frac{\psi(t) \psi\left(b_{0}\right)^{k}}{\psi\left(a_{0}\right)^{k}}=\frac{\psi(u) \psi\left(b_{0}\right)^{l}}{\psi\left(a_{0}\right)^{l}}=\tilde{\psi}\left(u x_{0}^{l}\right) .
$$

Thus $\tilde{\psi}$ is well-defined. Since $a_{0} \mid b_{0}$, we have $\left|\psi\left(a_{0}\right)\right| \geqq\left|\psi\left(b_{0}\right)\right|$. Hence $\left|\tilde{\psi}\left(t x_{0}^{k}\right)\right| \leqq|\psi(t)| \leqq 1$ for $t x_{0}^{k} \in T_{1}$; that is, $\tilde{\psi}$ is bounded. It is easy to show that $\tilde{\psi}$ extends $\psi$ and is multiplicative.

We now show that $\tilde{\psi}$ satisfies (A) on $T_{1}$. Suppose that $t x_{0}^{k} \mid u x_{0}^{l}$, $t, u \in T_{0}$. Then $t x_{0}^{k} r=u x_{0}^{l}$ for some $r \in G$. Hence $a_{0}^{k+l} t x_{0}^{k} r=a_{0}^{k+l} u x_{0}^{l}$ or $b_{0}^{k} a_{0}^{l} t r=b_{0}^{l} a_{0}^{k} u$ so that $\left|\psi\left(b_{0}\right) \psi\left(a_{0}\right)^{k} \psi(u)\right| \leqq\left|\psi\left(b_{0}\right)^{k} \psi\left(a_{0}\right)^{l} \psi(t)\right|$. It follows that

$$
\begin{aligned}
\left|\tilde{\psi}\left(u x_{0}^{l}\right)\right| & =\left|\frac{\psi(u) \psi\left(b_{0}\right)^{l}}{\psi\left(a_{0}\right)^{l}}\right|=\left|\frac{\psi(u) \psi\left(b_{0}\right)^{l} \psi\left(a_{0}\right)^{k}}{\psi\left(a_{0}\right)^{k+l}}\right| \leqq\left|\frac{\psi\left(b_{0}\right)^{k} \psi\left(a_{0}\right)^{l} \psi(t)}{\psi\left(a_{0}\right)^{k+l}}\right| \\
& =\left|\frac{\psi\left(b_{0}\right)^{k} \psi(t)}{\psi\left(a_{0}\right)^{k}}\right|=\left|\tilde{\psi}\left(t x_{0}^{k}\right)\right| .
\end{aligned}
$$

CAse 2. Suppose that Case 1 fails but that for some $x_{0} \in G-T_{0}$ and some $k_{1} \geqq 2$, we have $x_{0}^{k_{1}} \in T_{0}$ and $\psi\left(x_{0}^{k_{1}}\right) \neq 0$.

Let $k_{0}$ be the least positive integer such that $x_{0}^{k_{0}} \in T_{0}$. Since $x_{0}^{k_{0}} \mid x_{0}^{k_{1}}$, we have $\psi\left(x_{0}^{k_{0}}\right) \neq 0$. The denial of Case 1 is

$$
a x=b, a, b \in T_{0}, \text { and } x \in G-T_{0} \text { imply } \psi(b)=0 .
$$

Let $\alpha$ be any $k_{0}$ th root of $\psi\left(x_{0}^{k_{0}}\right)$; that is, any complex number such that $\alpha^{k_{0}}=\psi\left(x_{0}^{k_{0}}\right)$. Note that $T_{1}=\left\{t x_{0}^{k}: t \in T_{0}, 0 \leqq k<k_{0}\right\}$. Let $\tilde{\psi}\left(t x_{0}^{k}\right)$ $=\psi(t) \alpha^{k}$ for $t x_{0}^{k} \in T_{1}, 0 \leqq k<k_{0}$.

To see that $\tilde{\psi}$ is well-defined, we first suppose that $t x_{0}^{\boldsymbol{k}}=u x_{0}^{\mathbf{k}}$ where $t, u \in T_{0}, 0 \leqq k<k_{0}$. Then $t x_{0}^{k_{0}}=u x_{0}^{k_{0}}$. Hence $\psi(t) \psi\left(x_{0}^{k_{0}}\right)=\psi(u) \psi\left(x_{0}^{k_{0}}\right)$ so that $\psi(t)=\psi(u)$. Consequently, $\tilde{\psi}\left(t x_{0}^{k}\right)=\tilde{\psi}\left(u x_{0}^{k}\right)$. Suppose now that $t x_{0}^{k}=u x_{0}^{l}$ where $t, u \in T_{0}, 0 \leqq k<l<k_{0}$. Then $t x_{0}^{k+k_{0}-l}=u x_{0}^{k_{0}}$. By the minimality of $k_{0}, x_{0}^{k+k_{0}-l} \in T_{0}$. Thus by (B), $\psi\left(u x_{0}^{k_{0}}\right)=0$ and hence $\psi(u)=0$. We also have $t x_{0}^{k_{0}}=u x_{0}^{k_{0}} x_{0}^{l-z}$. By the minimality of $k_{0}$, $x_{0}^{l-k}-t T_{0}$ and hence by $(\mathrm{B}), \psi\left(t x_{0}^{k_{0}}\right)=0$. Thus $\psi(t)=0$. Hence $\tilde{\psi}\left(t x_{0}^{k}\right)$ 
$=\tilde{\psi}\left(u x_{0}^{l}\right)=0$. It is routine to verify that $\tilde{\psi}$ is bounded, extends $\psi$, and is multiplicative.

We now check that $\tilde{\psi}$ satisfies (A) on $T_{1}$. Suppose that $t x_{0}^{k} \mid u x_{0}^{l}$, $t, u \in T_{0}, 0 \leqq k<k_{0}, 0 \leqq l<k_{0}$. Then for some $r \in G, t x_{0}^{\boldsymbol{k}} r=u x_{0}^{l}$. Hence $t^{k_{0}} x_{0}^{k_{0}{ }^{k}} r^{k_{0}}=u^{k_{0}} x_{0}^{k_{0} l}$ so that $\left|\psi(u)^{k_{0}} \psi\left(x_{0}^{k_{0}}\right)^{l}\right| \leqq\left|\psi(t)^{k_{0}} \psi\left(x_{0}^{k_{0}}\right)^{k}\right|$ or $\left|\psi(u)^{k_{0}} \alpha^{k_{0} l}\right|$ $\leqq\left|\psi(t)^{k_{0}} \alpha^{k_{0} k}\right|$. Then $\left|\psi(u) \alpha^{l}\right| \leqq\left|\psi(t) \alpha^{k}\right|$, i.e. $\left|\tilde{\psi}\left(u x_{0}^{l}\right)\right| \leqq\left|\tilde{\psi}\left(t x_{0}^{k}\right)\right|$.

CASE 3. Suppose that Cases 1 and 2 fail but that for some

$$
x_{0} \in G-T_{0} \text { and } y_{0} \in G,
$$

we have $x_{0} y_{0} \in T_{0}$ and $\psi\left(x_{0} y_{0}\right) \neq 0$.

The denials of Cases 1 and 2 are (B) and

$$
x \in G-T_{0}, k \geqq 2, \quad \text { and } \quad x^{k} \in T_{0} \text { imply } \psi\left(x^{k}\right)=0 .
$$

Let $\alpha=\sup \left\{\left|\psi\left(x_{0}^{k_{y}}\right)\right|^{1 / k}: k \geqq 1, y \in G, x_{0}^{k} y \in T_{0}\right\}$; clearly $0<\alpha \leqq 1$. Let $\tilde{\psi}\left(t x_{0}^{k}\right)=\psi(t) \alpha^{k}$ for $t x_{0}^{k} \in T_{1}$.

To see that $\tilde{\psi}$ is well-defined, we first suppose that $t x_{0}^{k}=u x_{0}^{k}$, $t, u \in T_{0}, k \geqq 0$. Then $t x_{0}^{k} y_{0}^{k}=u x_{0}^{k} y_{0}^{k}$ and hence $\psi(t) \psi\left(x_{0} y_{0}\right)^{k}=\psi(u) \psi\left(x_{0} y_{0}\right)^{k}$. Thus $\psi(t)=\psi(u)$ and, consequently, $\tilde{\psi}\left(t x_{0}^{k}\right)=\tilde{\psi}\left(u x_{0}^{k}\right)$. Suppose now that $t x_{0}^{k}=u x_{0}^{l}, t, u \in T_{0}, 0 \leqq k<l$. Then $t x_{0}^{k} y_{0}^{k}=u x_{0}^{k} x_{0}^{l-k} y_{0}^{k}$. If $x_{0}^{l-k} \in T_{0}$, then by (C), $\psi\left(x_{0}^{l-k}\right)=0$ and hence $\psi(t) \psi\left(x_{0} y_{0}\right)^{k}=0$. If $x_{0}^{l-k} \in T_{0}$, then by (B), $\psi(t) \psi\left(x_{0} y_{0}\right)^{k}=0$. Hence in either case, $\psi(t)=0$. Since $t x_{0}^{k} y_{0}^{l}$ $=u x_{0}^{l} y_{0}^{l}$, we have $\left|\psi(u) \psi\left(x_{0} y_{0}\right)^{l}\right| \leqq|\psi(t)|=0$. Hence $\psi(u)=0$. Thus $\tilde{\psi}\left(t x_{0}^{k}\right)=\tilde{\psi}\left(u x_{0}^{l}\right)=0$. It is easy to verify that $\tilde{\psi}$ is bounded, extends $\psi$, and is multiplicative.

We now show that $\tilde{\psi}$ satisfies (A) on $T_{1}$. Suppose that $t x_{0}^{k} \mid u x_{0}^{l}$, $t, u \in T_{0}$. Then $t x_{0}^{k} r=u x_{0}^{l}$ for some $r \in G$. Suppose that $k=l$. Then $t x_{0}^{k} y_{0}^{k} r=u x_{0}^{k} y_{0}^{k}$ and hence $\left|\psi(u) \psi\left(x_{0} y_{0}\right)^{k}\right| \leqq\left|\psi(t) \psi\left(x_{0} y_{0}\right)^{k}\right|$ so that $|\psi(u)| \leqq|\psi(t)|$. Therefore $\left|\tilde{\psi}\left(u x_{0}^{k}\right)\right| \leqq\left|\tilde{\psi}\left(t x_{0}^{k}\right)\right|$.

Suppose that $k>l$. It follows that $t x_{0}^{k-l} r\left(x_{0} y_{0}\right)^{l}=u\left(x_{0} y_{0}\right)^{l}$. If $x_{0}^{k-l} r \notin T_{0}$, then by (B), $\psi(u) \psi\left(x_{0} y_{0}\right)^{l}=0$. Thus $\psi(u)=0$ and hence $\left|\tilde{\psi}\left(u x_{0}^{l}\right)\right|=0 \leqq\left|\tilde{\psi}\left(t x_{0}^{k}\right)\right|$. Suppose that $x_{0}^{k-l} r \in T_{0}$. Then $\left|\psi\left(x_{0}^{k-l} r\right)\right|^{1 / k-l}$ $\leqq \alpha$ or $\left|\psi\left(x_{0}^{k-l} r\right)\right| \leqq \alpha^{k-l}$. We then have

$$
\begin{aligned}
\left|\tilde{\psi}\left(u x_{0}^{l}\right) \psi\left(x_{0} y_{0}\right)^{l}\right| & =\left|\psi(u) \alpha^{l} \psi\left(x_{0} y_{0}\right)^{l}\right|=\left|\psi(t) \psi\left(x_{0}^{k-l} r\right) \psi\left(x_{0} y_{0}\right)^{l} \alpha^{l}\right| \\
& \leqq\left|\psi(t) \alpha^{k-l} \alpha^{l} \psi\left(x_{0} y_{0}\right)^{l}\right|=\left|\tilde{\psi}\left(t x_{0}^{k}\right) \psi\left(x_{0} y_{0}\right)^{l}\right|,
\end{aligned}
$$

from which it follows that $\left|\tilde{\psi}\left(u x_{0}^{l}\right)\right| \leqq\left|\tilde{\psi}\left(t x_{0}^{k}\right)\right|$.

Finally, suppose that $k<l$. Assume that $\left|\tilde{\psi}\left(t x_{0}^{k}\right)\right|<\left|\tilde{\psi}\left(u x_{0}^{l}\right)\right|$. Then $\left|\psi(t) \alpha^{k}\right|<\left|\psi(u) \alpha^{l}\right|$ or $|\psi(t)|^{1 / l-k} /|\psi(u)|^{1 / l-k}<\alpha$. Choose $m \geqq 1$ and $y \in G$ such that $x_{0}^{m} y \in T_{0}$ and $\left|\psi\left(x_{0}^{m} y\right)\right|^{1 / m}>|\psi(t)|^{1 / l-k} /|\psi(u)|^{1 / l-k}$. It follows that $\left|\psi\left(x_{0}^{m} y\right)^{l-k} \psi(u)^{m}\right|>\left|\psi(t)^{m}\right|$ or 


$$
\left|\psi\left(x_{0}^{m} y\right)^{l} \psi(u)^{m}\right|>\left|\psi(t)^{m} \psi\left(x_{0}^{m} y\right)^{k}\right| .
$$

Now $t x_{0}^{k} r=u x_{0}^{l}$ implies that $t^{m} x_{0}^{k m} r^{m} y^{k} y^{l-k}=u^{m} x_{0}^{l m} y^{l}$ so that $t^{m}\left(x_{0}^{m} y\right)^{k} \mid u^{m}\left(x_{0}^{m} y\right)^{l}$. Now applying (A) to $t^{m}\left(x_{0}^{m} y\right)^{k}, u^{m}\left(x_{0}^{m} y\right)^{l} \in T_{0}$, we get

$$
\left|\psi(u)^{m} \psi\left(x_{0}^{m} y\right)^{l}\right| \leqq\left|\psi(t)^{m} \psi\left(x_{0}^{m} y\right)^{k}\right|
$$

which contradicts (1). Hence $\left|\tilde{\psi}\left(u x_{0}^{l}\right)\right| \leqq\left|\tilde{\psi}\left(t x_{0}^{k}\right)\right|$.

CASE 4. Suppose that Cases 1, 2, and 3 fail.

The denial of Cases 1, 2, and 3 is

(D) $x \in G-T_{0}, y \in G$, and $x y \in T_{0}$ imply $\psi(x y)=0$.

Let $x_{0} \in G-T_{0}$ be arbitrary and fixed and let

$$
\tilde{\psi}\left(t x_{0}^{k}\right)=\left\{\begin{array}{ll}
\psi(t) & \text { for } k=0 \\
0 & \text { for } k \geqq 1
\end{array} \text { for } t x_{0}^{k} \in T_{1} .\right.
$$

We first check that $\tilde{\psi}$ is well-defined. Suppose that $t x_{0}^{\boldsymbol{k}}=u x_{0}^{l}, t, u \in T_{0}$, $0 \leqq k \leqq l$. If $k=l=0$, then clearly $\tilde{\psi}(u)=\tilde{\psi}(t)$. If $k>0$, then $\tilde{\psi}\left(t x_{0}^{k}\right)$ $=\tilde{\psi}\left(u x_{0}^{l}\right)=0$. If $k=0$ and $l>0$, then $t=u x_{0}^{l}=u x_{0}^{l-1} x_{0} \in T_{0}$. By (D), $\psi(t)=0$ and hence $\tilde{\psi}(t)=\tilde{\psi}\left(u x_{0}^{l}\right)=0$. It is routine to check that $\tilde{\psi}$ is bounded, extends $\psi$, and is multiplicative.

We show finally that $\tilde{\psi}$ satisfies (A) on $T_{1}$. Suppose that $t x_{0}^{k} \mid u x_{0}^{l}$, $t, u \in T_{0}$. Clearly $\left|\tilde{\psi}\left(u x_{0}^{l}\right)\right|=0 \leqq\left|\tilde{\psi}\left(t x_{0}^{k}\right)\right|$ unless $l=0$, so we may suppose that $t x_{0}^{k} \mid u$. Clearly $|\psi(u)| \leqq|\psi(t)|$ if $k=0$. Suppose that $k>0$. Then $t x_{0}^{k} r=u$ for some $r \in G$. Since $\left(t r x_{0}^{k-1}\right) x_{0} \in T_{0}$, we have by (D) that $\psi(u)=\psi\left(\operatorname{tr} x_{0}^{k}\right)=0$. Thus $\left|\tilde{\psi}\left(u x_{0}^{b}\right)\right|=0 \leqq\left|\tilde{\psi}\left(t x_{0}^{k}\right)\right|$.

This completes the proof.

REMARKs. Our theorem may be stated as follows, where $H$ is the complex unit disk.

Let $G$ be a commutative semigroup and let $S \subseteq G$ be a subsemigroup. Then if $f$ is a homomorphism of $S$ into $H$, the condition

$$
a \mid b \quad \text { and } a, b \in S \text { imply } f(a) \mid f(b),
$$

is necessary and sufficient for $f$ to be extendable to a homomorphism of $G$ into $H$.

A similar result is clearly valid if $H$ is $[0,1],[0, \infty$ [, or the entire complex plane. It is evident that the statement is not valid if $H$ is a semigroup that does not have a zero or identity or is not divisible. The proof of Case 3 together with the following example shows that some sort of completeness property is needed for $H$ in addition to divisibility and the existence of a zero and identity. Let $G=[0,1]$, 
$S=H=\{a \in[0,1]: a$ is algebraic $\}$, and $f$ be the identity map. It can be shown that $f$ cannot be extended to $G$. Our theorem thus cannot be extended to the case in which $H$ is a divisible commutative semigroup with zero and identity.

\section{REFERENCES}

1. E. Hewitt and H. S. Zuckerman, Finite dimensional convolution algebras, Acta Math. vol. 93 (1955) pp. 67-119.

2. - The $l_{1}$-algebra of a commutative semigroup, Trans. Amer. Math. Soc. vol. 83 (1956) pp. 70-97.

3. L. Pontrjagin, The theory of topological commutative groups, Ann. of Math. (2) vol. 35 (1934) pp. 361-388.

4. S. Schwarz, The theory of characters of finite commutative semigroups, Czechoslovak Math. J. vol. 4 (1954) pp. 219-247 (Russian).

5. - The theory of characters of commutative Hausdorff bicompact semigroups, Czechoslovak Math. J. vol. 6 (1956) pp. 330-361.

6. A. Weil, L'Integration dans les groupes topologiques et ses applications, Paris, Hermann et Cie., 1940.

UNIVERSITY OF WASHINGTON 